ARTIGO ORIGINAL ORIGINAL ARTICLE

Palavras-chave:

pessoa com deficiência física, necessidades, custos

\section{Custos adicionais da pessoa com deficiência física - São Paulo e Brasil}

\author{
Additional costs of living for people with physical \\ disabilities - São Paulo and Brazil
}

Paula Yuri Sugishita Kanikadan', Tania Yuka Yuba², Izabel de Loureiro Maior³, Fernanda Gabriela Borger ${ }^{4}$, Antonio Carlos Coelho Campino ${ }^{5}$

DOI: 10.21115/JBES.v11.n1.p26-33

\begin{abstract}
RESUMO
Objetivo: Calcular os custos adicionais da pessoa com deficiência física e investigar o seu impacto na renda familiar. Métodos: Seguindo a Classificação Internacional de Funcionalidade, identificaram-se quatro perfis de deficiência com graus diferentes de severidade quanto à mobilidade dos membros inferiores e superiores. Foi construída uma matriz de necessidades para estimar os custos adicionais, seguindo o Método de Padrão Orçamentário, a partir de grupos focais. Dados foram coletados em 2013. Utilizou-se o salário mínimo nacional de 2015 (R\$ 816,52) para estimar os custos. Resultados: Os custos adicionais variaram de 2 a 14 vezes o salário mínimo nacional. O perfil de menor custo adicional foi o 2, seguido do 1, 3 e 4 . O perfil 4 foi o de maior custo adicional devido à grande incapacidade das pessoas. Há exigência de alta intensidade de assistência pessoal e de oferta de equipamentos. Na assistência pessoal, calculou-se o trabalho dos cuidadores dividindo-o por um período de 2, 4, 8 e 24 horas, para São Paulo, Rio de Janeiro, Santa Catarina, Paraná e Brasil. Os custos com assistência pessoal são relevantes no total dos custos e variam conforme a região. Conclusões: Demonstrou-se que há um custo adicional para as pessoas com deficiência física em todos os perfis. Há risco de empobrecimento porque esses custos absorvem a renda dessas pessoas e de sua família, a qual não pode gastar em outros serviços. A renda pode ser, assim, insuficiente para as necessidades primordiais, ficando clara a necessidade de políticas públicas para prover serviços necessários ou benefícios financeiros.
\end{abstract}

\section{Keywords:}

people with physical

disabilities, needs, costs

\begin{abstract}
Objective: To calculate the additional costs of living for people with physical disabilities and to investigate their impact on family income. Methods: We identified four profiles of deficiency with different grades of severity, according to the International Classification of Functioning, Disability and Health. They were related to the extent of mobility of upper and lower limbs. Additional costs were evaluated through focal groups following the Budgetary Pattern Method. We also set up a matrix to express the additional costs. Data were collected in 2013. The national minimum wage from 2015 ( $R \$ 816,52)$ was considered to estimate the proportional costs. Results: Additional costs fluctuated between 2 to 14 times the national minimum wage. The profile with the minor additional cost was 2 , succeeded by 1, 3 and 4. Profile 4 showed the highest additional cost since people with physical disabilities need huge intensity of personal assistance and equipment supply. In personal assistance, we calculated the work of caregivers by sharing it into 2, 4, 8 and 24 hours/day. Costs with personal assistance diversify amongst São Paulo, Rio de Janeiro, Santa Catarina, Paraná and Brazil due to distinct minimum wage. Conclusions: This research showed the presence of an additional cost for people
\end{abstract}

Recebido em: 13/12/2018. Aprovado para publicação em: 28/03/2019

1. Professora do Curso de Medicina, Departamento de Ciências Naturais, Universidade Estadual do Sudoeste da Bahia, Vitória da Conquista, BA, Brasil.

2. Doutoranda do Departamento de Medicina Preventiva, Universidade de São Paulo, São Paulo, SP, Brasil.

3. Professora aposentada da Faculdade de Medicina da Universidade Federal do Rio de Janeiro, RJ, Brasil. Ex-secretária nacional de Promoção dos Direitos da Pessoa com Deficiência/Secretaria de Direitos Humanos, PR, Brasil.

4. Professora e coordenadora do Curso de Gestão Estratégica em Sustentabilidade da Fundação Instituto de Administração, pesquisadora da Fundação Instituto de Pesquisas Econômicas, São Paulo, SP, Brasil.

5. Professor sênior do Departamento de Economia, Faculdade de Economia, Administração e Contabilidade, Universidade de São Paulo, São Paulo, SP, Brasil.

Instituição onde o trabalho foi executado: Fundação Instituto de Pesquisas Econômicas (FIPE).

Informações sobre auxílios: Financiamento da Secretaria dos Direitos da Pessoa com Deficiência do Governo do Estado de São Paulo.

Congressos onde o estudo foi apresentado: 54th ERSA Congress, 26 a 29 de agosto de 2014, em São Petersburgo, Rússia. Autor correspondente: Paula Yuri Sugishita Kanikadan. Colegiado de Medicina. Estrada Bem Querer, Candeias, Vitória da Conquista, BA, Brasil. CEP: 3293-3391. Telefone: (77) 3425-9352. E-mail: pybrazil@gmail.com 
with physical disabilities in all profiles. There is a risk of impoverishment with sacrifice in family's income since they cannot waste in other domestic services. Thus, it is clear that there is a strong need of public policies to underpin these families with more health services or financial benefit.

\section{Introdução}

A Organização Mundial da Saúde (OMS) conceitua a deficiência como uma incapacidade inerente à natureza humana, ressaltando que quase todas as pessoas apresentarão, temporária ou permanentemente, incapacidades em algum momento de suas vidas, e aqueles que sobrevivem à terceira idade vivenciarão um aumento nas dificuldades de execução das atividades do dia a dia (OMS, 2011). Com efeito, pessoas com deficiência (PcD) enfrentam situações de desigualdade social e econômica, sujeitas a desvantagens na renda, na inserção no mercado de trabalho e na sua cidadania de forma geral. Um estudo da OMS aponta que famílias com PcD gastam relativamente mais com serviços de saúde do que famílias sem PCD (OMS, 2011, p. 10).

De fato, as PcD possuem necessidades diferentes, com intensidades variáveis, acometidas diferentemente nos diversos ciclos de vida. As necessidades das pessoas com deficiência física (PcDF) que impactam o custo são a assistência pessoal e os serviços de profissionais especializados na área de deficiência e de tecnologia assistiva. A intensidade das necessidades varia conforme o grau da deficiência e o ciclo de vida.

A mudança de conceituação de deficiência é de extrema importância ao deslocar o foco que, ao invés de ser a limitação de cada pessoa, passa a ser resultante das possibilidades de acesso dessa pessoa aos bens e aos serviços que a sociedade disponibiliza a todos.

O arcabouço teórico da Classificação Internacional de Funcionalidade (CIF), que representa um marco conceitual em matéria de deficiência, descreve os impedimentos corporais e a avaliação das barreiras sociais e de participação social, sendo a pessoa com deficiência não simplesmente um corpo com tais impedimentos, mas uma pessoa com impedimentos vivendo em um ambiente com barreiras. Essa abordagem está em consonância com o paradigma da deficiência como um prejuízo funcional resultante da interação complexa entre a estrutura e o funcionamento do corpo do indivíduo com deficiência e o ambiente em que ele está inserido, com limitação de atividade e restrição de participação da vida em sociedade.

Os custos de vida da PcDF têm sido considerados como um grande impedimento do acesso dessas pessoas ao cotidiano em sociedade, as quais possuem extrema dificuldade de adquirir tecnologias em saúde para ter uma qualidade de vida coerente com sua deficiência (Portugal et al., 2010; Smith et al., 2004).

A importância dos custos reforça-se quando se observam os dados mundiais de PcD. O relatório mundial sobre deficiência da OMS estima que 15,3\% da população mundial, aproximadamente 975 milhões de pessoas (dados de 2011'1), possuem algum tipo de deficiência, seja ela grave ou moderada (2011). No Brasil, segundo a metodologia do Censo do ano de $2000^{2}$ do Instituto Brasileiro de Geografia e Estatística (IBGE), que abrangeu graus diferentes de limitação funcional, o resultado foi de 24,5 milhões de pessoas ou $14,5 \%$ do total dos brasileiros ), ao passo que no Censo 2010 o IBGE computou 45,6 milhões de pessoas ou 23,9\% da população. Recentemente, o IBGE (2018) realizou uma releitura dos dados de PCD no Censo Demográfico 2010 à luz das recomendações do Grupo de Washington, e o resultado foi um quantitativo de 12.748 .663 pessoas, ou 6,7\% do total da população registrado pelo Censo Demográfico $2010^{3}$.

Este artigo, portanto, pretende analisar o impacto do valor do salário mínimo no custo adicional para as PCDF, considerando os diferentes salários vigentes no país e em alguns estados (São Paulo, Rio de Janeiro, Santa Catarina e Paraná). O presente estudo baseou-se no levantamento das necessidades adicionais e nos custos econômicos associados à deficiência física pela perspectiva das próprias PCDF e de suas famílias.

O texto está organizado em cinco partes: na primeira, fez-se sucinta introdução sobre o estado da arte da deficiência física; na sequência, descreve-se o percurso metodológico do estudo, considerando as metodologias atualmente reconhecidas na estimativa dos custos adicionais da deficiência física; nas terceira e quarta partes, respectivamente, expõem-se os principais resultados e discutem-se as evidências encontradas à luz dos mais relevantes marcos teóricos; por fim, a última parte tece algumas conclusões que podem auxiliar novas perspectivas no campo dos estudos da deficiência física.

\section{Métodos}

Utilizou-se a abordagem de padrão orçamentário, que possui como pressuposto o foco nas necessidades e nas informações que são obtidas por meio de técnicas de pesquisa qualitativa. O método consiste em levantar tudo o que é consumido em termos de bens e de serviços por uma pessoa com determinado perfil de deficiência e, a partir deste, calcular os custos. Para

\footnotetext{
Disponível em: http://apps.who.int/iris/bitstream/handle/10665/70670/ WHO_NMH_VIP_11.01_eng.pdf;isessionid=0DD12A9DEB3DC08E1B46F53C62052899? sequence=1. Acesso em: 24 out. 2018.

2 Disponível em: https://ww2.ibge.gov.br/home/presidencia/noticias/ 20122002censo.shtm. Acesso em 25 de abril de 2019.

3 Disponível em: ftp://ftp.ibge.gov.br/Censos/Censo_Demografico_2010/ metodologia/notas_tecnicas/nota_tecnica_2018_01_censo2010.pdf. Acesso em 24/10/2018.
} 
isso, foram utilizados os grupos focais (focus groups), os quais reuniram indivíduos com necessidades semelhantes.

O estudo britânico mais conhecido a adotar essa metodologia é Smith et al. (2004). Denominado Disabled people's cost of living - more than you would think, são analisados cinco perfis, sendo três para deficiências físicas (necessidades altas a médias, médias e baixas e intermitente) e dois para deficiências sensoriais (visual e auditiva). A mesma divisão é utilizada pelo DRC (Disability Resource Center) da Nova Zelândia em seu estudo sobre o custo da deficiência publicado em 2010. O estudo de Smith et al. (2004) teve como objetivo investigar as necessidades e os custos financeiros associados à deficiência pela perspectiva das próprias PCD.

Outro estudo que pode ser encaixado nessa metodologia é o de Portugal et al. (2010), que destaca a elaboração dos perfis de deficiência com base na Clasificación Internacional de Deficiencias, Discapacidades y Minusvalías. Nele, há o cruzamento de informações em dois eixos: (1) funções e estruturas do corpo e (2) atividades e participação. Com base nesses dois eixos, tem-se uma avaliação do custo da deficiência para cada tipo de perfil. Ao todo, os autores propõem dez perfis. Embora a divisão de grupos seja distinta da feita nos dois estudos supracitados, o trabalho também se desenvolve com base em três rodadas de grupos focais para cada perfil de interesse. Em adição e de forma complementar, há outras fontes de informações como entrevistas e grupos focais com especialistas, além de informações obtidas com membros de instituições especializadas.

Nesse sentido, a metodologia Padrão Orçamentário tem sido considerada a mais adequada para estimar os custos adicionais das PCD, sendo a mais utilizada por estudos recentes.

Com essa abordagem, determinaram-se as variáveis cruciais que influenciam os custos adicionais: o tipo de deficiência, o grau da deficiência, o ciclo de vida, o tipo de domicílio, as condições socioeconômicas e o nível de renda.

Para a definição dos tipos e graus de deficiência, seguimos o arcabouço da CIF para o levantamento das necessidades. A escolha implicou na articulação entre as dimensões referentes à estrutura do corpo afetada e os fatores ambientais. Essa interação dá origem a diferentes condições de funcionalidade que, por sua vez, determinam o grau de deficiência e suas respectivas necessidades associadas.

No campo da deficiência física, há uma complexidade muito maior de fatores envolvidos, tanto em termos de questões ligadas à saúde quanto daquelas ligadas às próprias estruturas do corpo diretamente afetadas e à multiplicidade de suas funções. Foi preciso aprofundar o conhecimento sobre a deficiência e levantar outros estudos e pesquisas para a definição dos perfis. Entre os estudos e pesquisas, decidiu-se utilizar a estrutura definida pelo grupo de Washington como base da composição dos perfis para a apuração de necessidades. $O$ Grupo de Washington sobre Estatísticas em Deficiência foi formado como resultado de um Seminário Internacional das Nações Unidas que ocorreu em Nova York, em junho de 2001.

Essa reunião resultou no reconhecimento de que um esforço estatístico e metodológico era necessário, em nível internacional para facilitar a comparação de dados sobre deficiência em diferentes países.

O processo de coleta de informações escolhido foi uma sequência de grupos focais. Na primeira rodada (grupo - "tarefa"), foi realizado um levantamento preliminar das necessidades pertinentes a cada perfil, seguida de outra rodada para a checagem e ajuste fino dessa lista de necessidades. Após essa etapa, montou-se a matriz de necessidades para a precificação.

A partir das necessidades levantadas pela pesquisa, os preços foram obtidos por coleta de campo e, no caso dos serviços, obtidos na base de dados da Pesquisa Nacional por Amostra de Domicílios (PNAD/2011) e da Pesquisa de Orçamentos Familiares (POF 2008/2009) para obter os preços de serviços e produtos, ambas do IBGE. São pesquisas com informações disponíveis em bancos de dados de "surveys", realizadas periodicamente, que têm como objetivo colher informações detaIhadas sobre as condições de vida e gastos da população, com base em amostras representativas.

As estimativas apuradas referem-se à população do estado de São Paulo e foram computadas em base mensal. Os valores foram convertidos pela taxa de câmbio dólar Paridade de Poder de Compra [Purchase Power Parity - PPP conversion factor private consumption (LCU per international \$), Banco Mundial (US\$ $1.00=R \$ 1,8494)$, dado de 2015].

Os valores do salário mínimo utilizados para estimar os custos da assistência pessoal são apresentados no quadro 1, a seguir.

Quadro 1. Valores do salário mínimo por região em dólar PPP (2015)

\begin{tabular}{ll}
\hline Localidade & Piso regional \\
\hline Brasil & $\$ 399,68$ \\
\hline São Paulo & $\$ 443,38$ \\
\hline Santa Catarina & $\$ 449,79$ \\
\hline Rio de Janeiro & $\$ 468,80$ \\
\hline Paraná & $\$ 521,64$ \\
\hline
\end{tabular}

\section{Custos para a deficiência física: a definição dos perfis de deficiência física}

Os perfis da deficiência física foram definidos usando graus de incapacidade funcional nos domínios mobilidade (eixo principal) e capacidade manipulativa dos membros superiores (eixo complementar). A combinação entre as quatro gradações das duas dimensões adotadas gerou 16 possibilidades (conforme figura 1). Essas 16 combinações abarcam todos os casos possíveis de comprometimento funcional nas duas dimensões escolhidas. 
Perfil 1 DA/DB: Caracteriza-se pelo grau de incapacidade da dimensão de mobilidade (não conseguem de modo algum caminhar ou subir degraus), com variações no comprometimento da dimensão da manipulação com os membros superiores (de nenhuma a alguma dificuldade para vestir-se e tomar banho).

Perfil 2 AD/BD: Caracteriza-se pelo grau de incapacidade com os membros superiores (não conseguem de modo algum realizar as atividades do autocuidado, como tomar banho ou se vestir), com variações no grau de mobilidade (de nenhuma a alguma dificuldade para caminhar ou subir degraus).

Perfil 3 CC/CB/BC: Caracteriza-se por uma junção de incapacidades nas duas dimensões: mobilidade (alguma ou grande dificuldade para caminhar e subir degraus) e capacidades manipulativas (alguma ou grande dificuldade para vestir-se ou tomar banho).

Perfil 4 DD/DC/CD: Caracteriza-se por uma junção de grandes incapacidades nas duas dimensões: grande dificuldade ou não conseguem de modo algum caminhar ou subir degraus; grande dificuldade ou não conseguem de modo algum tomar banho ou se vestir.

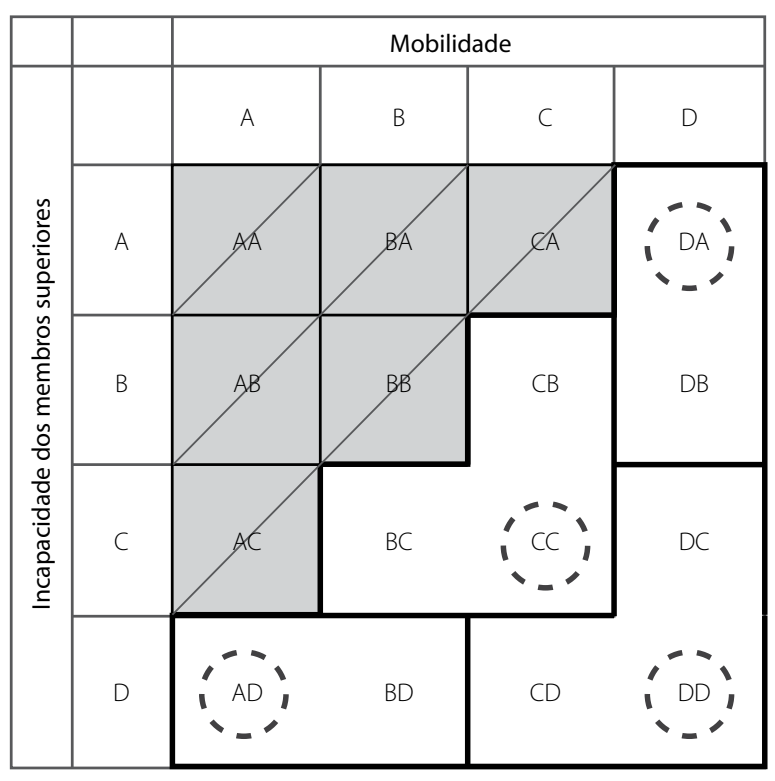

\section{MOBILIDADE}

Tem dificuldade permanente de caminhar ou

subir degraus?

A - Não, nenhuma dificuldade.

B - Sim, alguma dificuldade.

C - Sim, grande dificuldade.

D - Sim, não consegue de modo algum.

\section{CAPACIDADE MANIPULATIVA DOS MEMBROS SUPERIORES}

Tem dificuldade permanente de vestir-se ou

tomar banho?

A - Não, nenhuma dificuldade.

B - Sim, alguma dificuldade.

C - Sim, grande dificuldade.

D - Sim, não consegue de modo algum.

Figura 1. Perfis de deficiência física.
As necessidades das PCDF que acarretam custos econômicos diretos podem ser divididas entre assistência pessoal, serviços especializados, tecnologia assistiva e material de consumo. A figura 2 apresenta a matriz de necessidades de forma esquemática.

As grandes áreas de necessidades da matriz foram categorizadas por Atividades da Vida Diária - AVD (alimentar-se, vestir-se, higiene pessoal etc.), Atividades da Vida Prática - AVP (arrumar, cozinhar etc.), Comunicação (ler, escrever, digitar, telefonar), Mobilidade (deslocamento para trabalhar, lazer, escola) e Manutenção Funcional e Saúde (esta foi identificada pelos grupos de Deficiência Física).

Em cada uma dessas áreas, criou-se uma subdivisão a fim de suprir as necessidades das PCDF, que precisam de serviços de assistência pessoal, serviços especializados e tecnologia assistiva.

Na assistência pessoal, a PcDF precisa de um cuidador. Esse indivíduo seria um membro da família ou contratado, uma pessoa que auxilia a PCDF a realizar as atividades diárias, a acompanha em eventos externos, entre outras atividades.

Serviços Especializados foram definidos como o trabalho de profissionais de saúde e de educação que estão envolvidos no processo de reabilitação, aprendizagem e saúde, e manutenção funcional e saúde.

Tecnologia Assistiva foi compreendida como as adaptações no domicílio, como reformas e adequações domésticas, além da necessidade de equipamentos para autonomia e de material de consumo, produtos para higiene, saúde, entre outros, a fim de que haja garantia de acessibilidade às PCDF.

\section{Resultados}

Os resultados, representados na Tabela 1, apresentam os custos totais mensais por perfil e ciclo de vida em relação ao salário mínimo, considerando o piso regional do estado de São Paulo (R\$ 905,79 - dezembro de 2015).

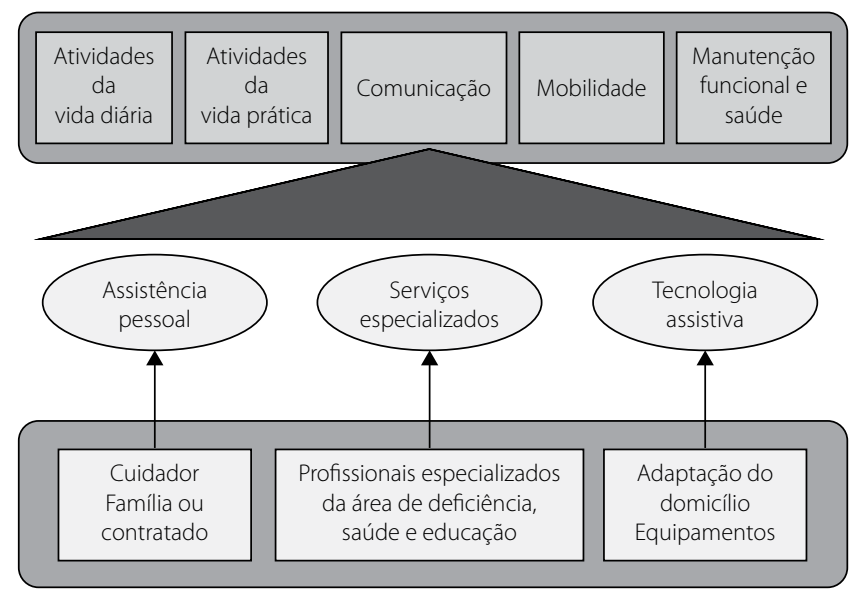

Figura 2. Matriz de necessidades. 
Tabela 1. Custos totais mensais em relação ao salário mínimo (em $\mathrm{R} \$$ 2015)

\begin{tabular}{lcccccccc}
\hline $\begin{array}{l}\text { Perfil } \\
\text { Ciclo de Vida }\end{array}$ & $\begin{array}{c}\text { Perfil 1 } \\
\text { DA,DB: } \\
\text { CT }^{*}\end{array}$ & $\begin{array}{c}\text { Perfil 1 } \\
\text { DA,DB: } \\
\text { CT/SM }\end{array}$ & $\begin{array}{c}\text { Perfil 2 } \\
\text { AD,BD: } \\
\text { CT }^{*}\end{array}$ & $\begin{array}{c}\text { Perfil 2 } \\
\text { AD,BD: } \\
\text { CT/SM }^{+}\end{array}$ & $\begin{array}{c}\text { Perfil 3 } \\
\text { CC,CB, BC: } \\
\text { CT }^{*}\end{array}$ & $\begin{array}{c}\text { Perfil 3 } \\
\text { CC,CB,BC: } \\
\text { CT/SM }^{+}\end{array}$ & $\begin{array}{c}\text { Perfil 4 } \\
\text { DD, CD,DC: } \\
\text { CT** }^{*}\end{array}$ & $\begin{array}{c}\text { Perfil 4 } \\
\text { DD, CD,DC: } \\
\text { CT/SM }^{*}\end{array}$ \\
\hline Infância/adolescência & 3260,58 & 3,6 & 1960,96 & 2,17 & 4025,9 & 4,44 & 12127,77 & 13,39 \\
\hline Jovem & 3042,96 & 3,4 & 1743,23 & 1,92 & 3807,97 & 4,2 & 11903,56 & 13,14 \\
\hline Adulto & 3054,65 & 3,4 & 1754,79 & 1,94 & 3819,28 & 4,22 & 11907,99 & 13,15 \\
\hline Idoso & 3160,47 & 3,5 & 1859,62 & 2,05 & 3921,84 & 4,33 & 9101,76 & 10,05 \\
\hline
\end{tabular}

* CT: custo total.

${ }^{+}$CT/SM: custo total sobre salário mínimo.

Nota-se que o perfil 1 DA, DB, definido como o grau de incapacidade com os membros inferiores e alguma dificuldade com os membros superiores, apresenta, para todos os ciclos de vida, custos totais semelhantes e, consequentemente, custos totais em relação ao salário mínimo também similares. Para a infância e adolescência, observa-se a razão CT/SM =3,60, um pouco maior que na fase do idoso (CT/SM = 3,50), ao passo que nos ciclos de vida jovem e adulto essa razão é praticamente igual $(3,40)$.

É interessante observar que o perfil $2 \mathrm{AD}, \mathrm{BD}$, caracterizado pelo grau de incapacidade com os membros superiores e alguma restrição na mobilidade dos membros inferiores, apresenta menor proporção CT/SM $(2,17 ; 1,92 ; 1,94 ; 2,05$, respectivamente, infância e adolescência; jovem; adulto; idoso) quando comparado com o perfil 1, em todos os ciclos de vida.

Conforme aumenta o grau de incapacidade da PcDF, cresce também a relação $C T / S M$. Com efeito, ao se analisar a relação CT/SM do perfil 3 CC, CB, BC, caracterizado como uma junção de incapacidades nas dimensões mobilidade e manipulativas, observa-se que essa razão praticamente se duplica nos quatro ciclos de vida $(4,44 ; 4,20 ; 4,22 ; 4,33$, respectivamente, infância e adolescência; jovem; adulto; idoso), se a confrontarmos com os valores obtidos para o perfil 2.

O perfil 4 DD, CD, DC, cuja PcDF demonstra grau de incapacidade bastante elevado nas duas dimensões, mostra a proporção CT/SM acima de 13 para os três primeiros ciclos de vida e de 10,05 para a fase do idoso. Os quatro ciclos de vida nesse perfil demonstram custos adicionais exacerbados e altos quando se comparam aos perfis 1, 2 e 3 .

Nessas condições, os quatro perfis de deficiência física demonstraram a necessidade de a PCDF de enfrentar custos adicionais em relação ao salário mínimo para viver em condições orçamentárias semelhantes às de uma pessoa sem deficiência física, nas três grandes áreas expressas na matriz de necessidades: serviços especializados, tecnologia assistiva e assistência pessoal.

Entre essas três áreas, os custos com assistência pessoal, para os quatro ciclos de vida, foram os que mais se destacaram como impedimento à PCDF de possuir qualidade de vida compatível com sua deficiência, uma vez que em todos os perfis de deficiência física a necessidade da assistência pessoal demonstrou-se muito mais intensa quando comparada não somente aos outros tipos de deficiência (visual, auditiva, intelectual), como também às próprias necessidades da PcDF de mais serviços especializados e de novas tecnologias assistivas.

A intensidade de trabalho do cuidador varia conforme o perfil da PCDF. Por esse motivo, foram consideradas a seguintes gradações: intensidade leve (2 horas por dia), moderada (4 horas por dia), intensa (8 horas por dia) e integral (24 horas por dia).

Com essas informações, calcularam-se os custos de assistência pessoal por perfil de deficiência não apenas para o estado de São Paulo, como também para outros estados do Brasil: Rio de Janeiro, Santa Catarina e Paraná, além do Brasil.

A Tabela 2 mostra os custos de assistência pessoal por perfil de deficiência física nessas regiões do Brasil, expressos em dólar. O salário considerado para o cálculo foi o piso salarial do Brasil e para os estados de São Paulo, Rio de Janeiro, Santa Catarina e Paraná. O custo é o salário ponderado pela intensidade da necessidade.

Observa-se que há pequenas diferenças entre os quatro estados, nos quatro perfis de deficiência física, quando se compara com o Brasil. Isso se justifica porque os pisos salariais de São Paulo (US\$ 443,38), Santa Catarina (US\$ 449,79) e Rio de Janeiro (US\$ 468,80) são muito próximos, tendo o Paraná um piso um pouco maior $($ US\$ 521,64) e o Brasil uma média de piso salarial inferior (US\$399,68) em relação aos demais estados.

Entretanto, quando se comparam os quatro perfis de deficiência física em todos os estados e no Brasil, nota-se uma diferença significativa nos custos adicionais de assistência pessoal, especialmente no grupo 4 DD, CD, DC, no qual existe grande limitação física nos membros superiores e inferiores da PcDF, o que requer um cuidador 24 horas por dia a um custo médio no Brasil de US\$ 1.089,35. Considerando que o piso salarial do Brasil é de US\$399,68, a PCDF necessitaria de 2,73 vezes o salário mínimo só para cobrir os custos de assistência pessoal. Esse valor repete-se para São Paulo, Santa Catarina, Rio de Janeiro e Paraná. 
Tabela 2. Custos de assistência pessoal por perfil e região em dólar PPP (2015)

\begin{tabular}{lcccc}
\hline Perfil & $\begin{array}{c}\text { Grupo 1 } \\
\text { DA, DB }\end{array}$ & $\begin{array}{c}\text { Grupo 2 } \\
\text { AD, BD }\end{array}$ & $\begin{array}{c}\text { Grupo 3 } \\
\text { CC, CB,BC }\end{array}$ & $\begin{array}{c}\text { Grupo 4 } \\
\text { DD, CD,DC }\end{array}$ \\
\hline Necessidades de assistência pessoal & 4 horas/dia & 2 horas/dia & horas/dia & 24 horas/dia \\
\hline Brasil & $\$ 181,56$ & $\$ 90,78$ & $\$ 363,12$ & $\$ 1.089,35$ \\
\hline São Paulo & $\$ 201,41$ & $\$ 100,70$ & $\$ 402,82$ & $\$ 1.208,45$ \\
\hline Santa Catarina & $\$ 204,33$ & $\$ 102,16$ & $\$ 408,65$ & $\$ 1.225,96$ \\
\hline Rio de Janeiro & $\$ 212,96$ & $\$ 106,48$ & $\$ 125,92$ & $\$ 1.277,77$ \\
\hline Paraná & $\$ 236,96$ & $\$ 118,48$ & $\$ 1.421,76$ & \\
\hline
\end{tabular}

Já o perfil 3 CC, CB, BC mostra valores de custo de um cuidador contratado durante 8 horas em um dia muito próximos dos valores máximos de piso salarial, por região: US\$ 363,12 para o Brasil é o custo de um profissional ou familiar que cuida de uma PcDF; o salário mínimo é de US\$ 399,68. Isso também é observado nas demais regiões do Brasil.

Finalmente, para o perfil 1 de deficiência física, nota-se que um cuidador custa um pouco menos que a metade do valor do salário mínimo (Brasil: US\$ 181,56; salário mínimo: US\$ 399,68), enquanto, para o perfil 2, contratar um cuidador significa que ele custará aproximadamente 1/4 do salário mínimo de cada região (Brasil: US\$ 90,78; salário mínimo: US\$ 399,68).

\section{Discussão}

As PcDF demonstraram a necessidade de três grandes elementos fundamentais, conforme expresso na matriz de necessidades: 1. serviços especializados, 2. tecnologia assistiva e 3. assistência pessoal.

Quanto aos serviços especializados, esses são importantes no processo de reabilitação e de manutenção funcional nas áreas de saúde, educação e terapias de reabilitação, a fim de que haja também manutenção física da PcDF. Por isso, os custos variam para as diferentes fases de vida da PcDF. Os custos, nessa fase, são diluídos ao longo da vida, sendo contínuos para a manutenção funcional. Os custos com tecnologia assistiva também variam conforme o ciclo de vida, considerando que a PCDF necessita de equipamentos e de determinadas adaptações no domicílio que variam ao longo da vida. Ademais, podem ocorrer mudanças no perfil de deficiência conforme a PCDF caminha para anos mais avançados de vida.

O destaque deu-se na necessidade de assistência pessoal, de forma que se observou, nos quatro perfis de deficiência física, que a necessidade da assistência pessoal é determinante e fundamental, pois, por mais habilidades que as PCDF tenham adquirido durante o processo de reabilitação, ou dos benefícios que os equipamentos e a adaptação domiciliar possam Ihes fornecer, elas terão sempre as restrições físicas que as impossibilitam de realizar algumas atividades.

De fato, no perfil 2 AD, BD, em que as habilidades para executar as atividades da vida diária são muito restritas, há sempre a necessidade do auxílio de outras pessoas para sua realização. No caso do perfil 4 DD, DC, CD, a assistência pessoal é imprescindível para a realização de praticamente todas as atividades da vida diária, prática e mobilidade.

Os resultados da pesquisa demonstraram que há um custo adicional para as PCD em todos os perfis estudados. Há risco de empobrecimento, porque esses custos absorvem a renda da PCD e de sua família; fica muito claro que, em casos extremos, como o do perfil 4 da deficiência física, o custo pode ultrapassar em várias vezes (14) a renda total das famílias. Mesmo para as famílias com rendimento total maior, superior a 10 SM, o custo pode absorver mais do que $100 \%$ da sua renda. Esse é um sacrifício da renda, porque a família não pode gastar em outros bens e serviços e tem que reduzir seu padrão de vida. A renda disponível pode ser, assim, insuficiente para satisfazer as necessidades dessas famílias. Fica claro que existe a necessidade de políticas públicas para dar suporte a essas famílias, provendo os serviços e equipamentos necessários ou por meio de benefícios financeiros.

Além dos custos adicionais de assistência pessoal, deve-se considerar outras dimensões às quais está exposta a PcDF, como o papel social do cuidador, especialmente se ele for um membro da família, consoante estudos de Trigueiro et al. (2011) e Madureira (2002), os quais reportaram as difíceis condições mentais e operacionais de familiares que cuidam da PcDF: há ausência de orientações e preparo emocional para que esses cuidadores possam lidar com as complicações da PCDF, que perpassam o cunho econômico, ocasionando grave estresse físico e emocional no cuidador, e, consequentemente, tais desgastes impactam diretamente no cuidado à PcDF.

Apesar de a Política Nacional de Saúde da PcD publicada em 2002 possuir como algumas de suas diretrizes a promoção da qualidade de vida, a atenção integral à saúde, a melhoria dos mecanismos de informação, a capacitação de recursos humanos e a organização dos serviços, dos quais o papel do cuidador seria fundamental dentro de todas essas diretrizes, nota-se muito pouco cuidado com a importante função do cuidador. Observa-se, de modo geral, que os serviços de saúde ainda traduzem a PCDF como aquela que só necessita de tecnologias relacionadas aos equipamentos fornecidos pelo Sistema 
Único de Saúde (SUS) e à reabilitação. Cruz (2012) vai adiante e argumenta que a PcDF, muitas vezes, acaba não adquirindo determinado equipamento no serviço público de saúde por desconhecimento ou receio no uso da própria tecnologia.

Se esse parece ser o cenário atual pelo qual passa a PCDF, salvo engano, pouco ou quase nada se trabalha com relação à necessidade de atenção especial aos cuidadores. Embora tenha sido criada a Rede de Cuidados à Pessoa com Deficiência (Brasil, 2012), ampliando o acesso e qualificando o atendimento às PCD no SUS, e também ampliando a integração e a articulação dos serviços de reabilitação na Atenção Básica, este estudo demonstrou que mais ações, em todos os âmbitos, podem ser pensadas para auxiliar na assistência pessoal da PCDF.

Por fim, o ciclo de vida é um fator que influencia as necessidades das PcDF. Entretanto, foi possível observar que as necessidades são as mesmas para os ciclos de vida jovem, adulto e idoso, embora o envelhecimento acentue as necessidades, levando à mudança de perfil de funcionalidade da PCDF, intensificando as dificuldades de mobilidade e/ou das capacidades manipulativas. Por exemplo, pessoas usuárias de muletas, com grande dificuldade de caminhar e subir degraus e nenhuma dificuldade em tomar banho ou vestir-se, à medida que envelhecem, têm maior desgaste físico, ficando mais vulneráveis aos acidentes e, consequentemente, perdendo a mobilidade; assim, mudam de perfil, muitas vezes se tornando pessoas usuárias de cadeira de rodas e, assim, não conseguem de modo algum caminhar ou subir degraus. Dessa forma, as suas necessidades também serão de outro perfil.

\section{Conclusões}

As diferenças regionais têm impacto significativo no custo adicional de vida das PCDF, porque elas dependem da assistência pessoal, serviço provido por um cuidador contratado ou da família, e não há políticas públicas ou serviços de apoio para atender a essa necessidade fundamental dessa modalidade de deficiência.

Os serviços especializados podem ser financiados ou providos pelo setor público: as PCDF que não dispõem de recursos são atendidas pelo SUS e/ou pelas entidades filantrópicas, com os riscos inerentes de filas e atrasos no atendimento, e as dificuldades no deslocamento por conta da distância, o que compromete a sua qualidade de vida e, consequentemente, sua independência e autonomia. Quando se tem à disposição serviços de saúde, transporte e educação, há melhora na independência e na autonomia das PCDF.

Entretanto, a assistência pessoal é necessária sempre e deve ser provida por um cuidador, membro da família ou contratado, e representa um custo significativo para a PcDF e sua família, sendo necessário refletir em políticas públicas efetivas para apoiar de alguma forma essa necessidade.

Em suma, a necessidade de arcar com os custos adicionais computados nessa pesquisa representa uma barreira à partici- pação na sociedade da PcDF, primeiro porque ela não possui renda suficiente para arcar com todas as despesas necessárias para viver com um mínimo de qualidade de vida e, em segundo lugar, porque ela fica limitada ao espaço físico e social ao qual consegue chegar.

\section{Agradecimentos}

Agradecemos imensamente a todas as pessoas que participaram dos grupos focais e aos profissionais que colaboraram na execução deste estudo.

\section{Referências bibliográficas}

Brasil. Ministério da Saúde. Política Nacional de Saúde da Pessoa com Deficiência. Série B: Textos Básicos de Saúde. Brasília, DF: 2010. 24p.

Brasil. Ministério da Saúde. Portaria no 793, de 24 de abril de 2012. Institui a Rede de Cuidados à Pessoa com Deficiência no âmbito do Sistema Único de Saúde. 2012.

Cruz DMC. Papéis ocupacionais e pessoas com deficiências físicas: independência, tecnologia assistiva e poder aquisitivo [tese]. São Carlos: Centro de Educação e Ciências Humanas da Universidade Federal de São Carlos; 2012. 230p.

IBGE - Instituto Brasileiro de Geografia e Estatística. Características da população e dos domicílios: resultados do universo. 2000.

IBGE - Instituto Brasileiro de Geografia e Estatística. Censo Demográfico 2000. Características gerais da população: resultados da amostra. Rio de Janeiro: IBGE; 2003.

IBGE - Instituto Brasileiro de Geografia e Estatística. Censo Demográfico 2010. Características gerais da população, religião e pessoas com deficiência. Rio de Janeiro: IBGE; 2012.

IBGE - Instituto Brasileiro de Geografia e Estatística. PNAD - Pesquisa Nacional por Amostra de Domicílios. IBGE; 2011.

IBGE - Instituto Brasileiro de Geografia e Estatística. POF - Pesquisa de Orçamentos Familiares: despesas, rendimentos e condições de vida. IBGE; 2008/2009.

IBGE - Instituto Brasileiro de Geografia e Estatística. Nota Técnica 01/2018. Releitura dos dados de pessoas com deficiência no Censo Demográfico 2010 à luz das recomendações do Grupo de Washington. Disponível em: ftp://ftp.ibge.gov.br/Censos/Censo_Demografico_2010/metodologia/ notas_tecnicas/nota_tecnica_2018_01_censo2010.pdf. Acesso em 22/04/2019.

Madureira NCM. O saber-fazer do cuidador familiar da pessoa com deficiência física: um estudo no pré- e trans-reabilitação [dissertação]. São Paulo: Escola de Enfermagem da Universidade de São Paulo; 2002. 75p.

OMS - Organização Mundial da Saúde. Relatório mundial sobre a deficiência. Tradução: Lexicus Serviços Linguísticos, Secretaria de Estado dos Direitos da Pessoa com Deficiência. São Paulo; 2011.

OMS - Organização Mundial da Saúde. Centro Colaborador da Organização Mundial da Saúde para a Família de Classificações Internacionais (Org.). Classificação internacional de funcionalidade, incapacidade e saúde. São Paulo: Editora da Universidade de São Paulo; 2003.

ONU - Organização das Nações Unidas. Convenção sobre os direitos das pessoas com deficiência e protocolo facultativo. Assembleia Geral das Nações Unidas; 2009.

Portugal S, Martins BS, Ramos LM, Hespanha P, Alves J, Fidalgo J. Estudo de avaliação do impacto dos custos financeiros e sociais da deficiência. Coimbra: Centro de Estudos Sociais; 2010. 
Smith N, Middleton S, Ashton-Brooks K, Cox L, Dobson B, Reith L. Disabled people's costs of living. More than you would think. Inglaterra: Joseph Rowntree Foundation; 2004.

Tibble M. Review on existing research on de extra costs of disability; a report of research carried out by the Department for Work and Pensions. Department for Work and Pensions. 2005. (Working Paper n. 21)
Trigueiro LCL, Aragão POR, Lemos MTM. Perfil sociodemográfico e índice de qualidade de vida de cuidadores de pessoas com deficiência física. Phys Ther Res. 2011;18(3):223-7.

Zaidi A, Burchardt T. Comparing incomes when needs differ: equalisation for the extra costs of disability in the UK. Review Income Wealth. 2005:51(1):89-114. 